\title{
Influência da densidade do solo infestado por nematoide no desenvolvimento inicial de cana-de-açúcar
}

\author{
Carmem C. M. de Sousa ${ }^{1}$, Elvira M. R. Pedrosa ${ }^{2}$, Mario M. Rolim $^{3}$, \\ João V. Pereira Filho ${ }^{4}$ \& Marcela A. L. M. de Souza ${ }^{5}$
}

${ }^{1}$ UFRPE. Recife, PE. E-mail: crismareco@hotmail.com

${ }^{2}$ UFRPE. Recife, PE. E-mail: elvira.pedrosa@dtr.ufrpe.br

${ }^{3}$ UFRPE. Recife, PE. E-mail: rolim@dtr.ufrpe.br (Autor correspondente)

${ }^{4}$ DEA/UFC. Fortaleza, CE. E-mail: joao_valdenor@hotmail.com

${ }^{5}$ UFRPE. Recife, PE. E-mail: marcelandrade lima@hotmail.com

\section{Palavras-chave:}

Saccharum

nematóide de galha

densidade do solo

\begin{abstract}
R E S U M O
Em áreas cultivadas com cana-de-açúcar no Nordeste do Brasil, a compactação do solo e a presença de altas densidades populacionais de nematoides, principalmente Meloidogyne spp. restringem severamente a produtividade agrícola. O presente estudo teve como objetivo avaliar o efeito de quatro níveis de densidade do solo infestado por nematoides da espécie $M$. incognita no desenvolvimento inicial da cana-de-açúcar, variedade RB 863129, em condições de casa de vegetação. As avaliações, realizadas 90 dias após a infestação do solo, se fundamentaram na altura da planta, diâmetro do colmo, número de folhas, biomassa fresca das raízes, biomassa fresca e biomassa seca da parte aérea, número de ovos do nematoide por grama de raiz. O aumento dos níveis de compactação do solo de 1,65 para $1,82 \mathrm{~kg} \mathrm{dm}^{-3}$ proporcionou redução na multiplicação de $M$. incognita e aumento no comprimento do colmo da cana-de-açúcar mas não afetou as outras variáveis de desenvolvimento da planta.
\end{abstract}

Key words:

Saccharum

root-knot nematode

soil density

\section{Influence of density of soil infested with nematode on initial growth of sugarcane}

\begin{abstract}
A B S T R A C T
In cultivated areas of sugarcane in Northeastern Brazil, soil compaction and infestation of nematodes, mainly Meloidogyne spp., severely restrict crop production. The present study aimed to evaluate the effect of four levels of density of soil infested with nematodes of the species $M$. incognita in the early development of sugarcane variety RB 863129 in greenhouse conditions. Evaluations were carried out 90 days after soil infestation, based on plant height, stalk diameter, number of leaves, root and shoot fresh biomass, shoot dry biomass and number of nematode eggs. The increase in soil compaction level from 1.65 to $1.82 \mathrm{~kg} \mathrm{dm}^{-3}$ decreased $M$. incognita reproduction and increased sugarcane stalk length, but it did not affect the other plant development variables.
\end{abstract}

\section{INTRODUÇÃo}

Atualmente, a cultura da cana-de-açúcar (Saccharum L.) se reveste de grande importância socioeconômica para o Brasil enquanto a aceitação internacional do etanol como combustível aliado à lucratividade alcançada pelo setor sucroalcooleiro, tem trazido euforia aos produtores de cana-de-açúcar, com reflexos na intensificação e na expansão do seu cultivo. Dentre os fatores de produção relacionados com a modernização da canavicultura brasileira, o crescimento da demanda pela colheita mecanizada se destaca intensificando o tráfego sobre o solo, muitas vezes em condições desfavoráveis em termos de conteúdo de água, tornando praticamente inevitável a ocorrência da compactação do solo (Iaia et al., 2006; Vicente, 2011).

A compactação, resultado do processo de aumento da densidade do solo e resistência à penetração, é uma das principais causas da degradação do solo. Assim, o tráfego de máquinas vem intensificando o processo de compactação em virtude de promover alterações nas propriedades físicas dos solos afetando diretamente a qualidade do solo e da água e trazendo, como consequência, alterações no crescimento e na produtividade da cultura, além do aumento dos processos erosivos (Bordin et al., 2005; Freddi et al., 2009). Por outro lado, a resposta das plantas à compactação varia de acordo com os solos, condições de concentração de água no solo, densidade do solo e resistência mecânica à penetração com o crescimento radicular, tipos de culturas e variedades (Silva et al., 2006).

Como outras culturas de grande valor econômico, além da alta vulnerabilidade à compactação do solo devido ao manejo, a cana-de-açúcar é susceptível a diferentes componentes da microbiota e microfauna do solo. Os nematoides, sobretudo Meloidogyne spp. provocam grandes perdas agrícolas que, em média, variam entre 20 e $40 \%$ da produtividade da massa foliar 
da concentração de açúcar no primeiro corte em variedades susceptíveis reduzindo, além da produtividade, a longevidade da cana soca (Campos et al., 2006). Entre as espécies de nematoide de maior importância para a cana-de-açúcar no Nordeste brasileiro se destaca a M. incognita, cujas reduções na produtividade podem variar de 36 a 77\% (Miranda et al., 2003).

Embora vários estudos tenham sido conduzidos para conhecer e caracterizar os nematoides parasitas da cana-deaçúcar no Brasil e o efeito de práticas culturais sobre a população desses organismos no solo (Miranda et al., 2003; Pedrosa et al., 2005; Matos et al., 2011), informações quanto à influência do nematoide na resposta da planta à compactação do solo, são escassas (Cardoso et al., 2012).

Ademais, a distribuição dos nematoides depende das características do solo no qual habita e que permite a orientação, movimentação e reprodução desses organismos, estando frequentemente relacionada com a distribuição de raízes, assim como com a estrutura, textura, umidade e temperatura do solo (Kimenju et al., 2009). Segundo Pattison et al. (2008), Mondino et al. (2009) e Cardoso et al. (2012), os nematoides apresentam grande potencial como bioindicadores de qualidade do solo devido à resposta rápida às mudanças no ambiente $\mathrm{e}$ ações de gestão do solo. Neste estudo objetivou-se avaliar o efeito de quatro níveis de compactação de solo infestado com $M$. incognita no desenvolvimento inicial de plantas de canade-açúcar.

\section{Material e Métodos}

O experimento foi conduzido em casa de vegetação na Universidade Federal Rural de Pernambuco ( $8^{\circ} 01^{\prime}$ 05" latitude sul, $34^{\circ} 56^{\prime} 48^{\prime \prime}$ latitude oeste e altitude de $4 \mathrm{~m}$ ) com temperatura média de $30,25^{\circ} \mathrm{C}$ e umidade relativa do ar de $77,38 \%$.

O solo utilizado foi um Argissolo Amarelo distrófico, segundo a EMBRAPA (1999), de textura franco-arenosa, coletado na camada de 0,50-0,90 m, município de Carpina, PE, cujas características químicas foram: $\mathrm{pH}$ (água) de 4,6; $\mathrm{Ca}^{2+} \mathrm{de}$ $0,37 \mathrm{cmol}_{\mathrm{c}} \mathrm{kg}^{-1} ; \mathrm{Mg}^{2+}$ de $0,51 \mathrm{cmol}_{\mathrm{c}} \mathrm{kg}^{-1} ; \mathrm{Na}^{+} 0,03 \mathrm{cmol}_{\mathrm{c}} \mathrm{kg}^{-1} ; \mathrm{K}^{+}$ $0,04 \mathrm{cmol}_{\mathrm{c}} \mathrm{kg}^{-1} ; \mathrm{H}^{+}+\mathrm{Al}^{3+} 2,84 \mathrm{cmol}_{\mathrm{c}} \mathrm{kg}^{-1} ; \mathrm{Al}^{3+} 0,91 \mathrm{cmol}_{\mathrm{c}} \mathrm{kg}^{-1}$; $\mathrm{S} 0,95 \mathrm{cmol}_{\mathrm{c}} \mathrm{kg}^{-1}$; T 4,70 $\mathrm{cmol}_{\mathrm{c}} \mathrm{kg}^{-1} ; \mathrm{C} 7,0 \mathrm{~g} \mathrm{~kg}^{-1} ; \mathrm{N} 0,3 \mathrm{~g} \mathrm{~kg}^{-1}$; M.O. $23 \mathrm{~g} \mathrm{~kg}^{-1}$; P assimilável $8 \mathrm{mg} \mathrm{kg}^{-1}$ e K $0,04 \mathrm{mg} \mathrm{dm}^{-3}$. Antes da montagem do experimento o material solo foi peneirado em peneira de $5 \mathrm{~mm}$ e autoclavado durante $1 \mathrm{~h}$ e $30 \mathrm{~min}$, por 2 dias consecutivos, no intervalo de $24 \mathrm{~h}$, na temperatura de 120 ${ }^{\circ} \mathrm{C}$ e pressão de $101 \mathrm{kPa}$.

A variedade de cana-de-açúcar usada foi a RB 863129, cedida pela Biofábrica do Centro de Tecnologias Estratégicas do Nordeste - CETENE, adaptada apenas ao cultivo de sequeiro. Após 20 dias de aclimatação em substrato na casa de vegetação as plantas foram transferidas para vasos com $70 \mathrm{~cm}$ de altura e $58 \mathrm{~cm}$ de diâmetro contendo o solo autoclavado, o qual recebeu calagem e adubação de fundação de NPK segundo UFC (1993). Após mais 30 dias no vaso as plantas foram expostas em delineamento inteiramente casualizado com cinco tratamentos (T1 - sem compactação com nematoide, T2 - 16\% compactação com nematoide; T3
- 48\% compactação com nematoide; T4 - 64\% compactação com nematoide e T5 - testemunha absoluta, sem compactação sem nematoide) que equivaleu as seguintes densidades do solo ( $\Delta$ ds) $1,65,1,69,1,78$ e $1,82 \mathrm{~kg} \mathrm{dm}^{-3}$, respectivamente, com cinco repetições cada.

O ensaio destinado à avaliação do efeito da compactação foi determinado a partir da análise granulométrica do solo utilizando ensaio de Proctor normal que seguiu o padrão normalizado pela ABNT (1986) obtendo-se a densidade máxima de $1,91 \mathrm{~kg} \mathrm{dm}^{-3} \mathrm{com} 11,03 \%$ de umidade. Determinouse a amplitude da variação da densidade do solo $(\Delta \mathrm{ds})$ de cada tratamento através da metodologia de Medeiros et al. (2005) com a seguinte expressão:

$\mathrm{ds}=$ densidade máxima $\left(1,91 \mathrm{~kg} \mathrm{dm}^{-3}\right)-$ a densidade do solo solto peneirado $\left(1,65 \mathrm{~kg} \mathrm{dm}^{-3}\right)=0,26 \mathrm{~kg} \mathrm{dm}^{-3}$

Em seguida, determinaram-se os demais níveis de densidades do solo:

nível 0: $0 \% \mathrm{da} \mathrm{ds}=\mathrm{a}$ densidade mínima do solo sem compactação $=1,65 \mathrm{~kg} \mathrm{dm}^{-3}$;

nível 1: $16 \%$ da ds e as demais densidades foram determinadas por regra de três simples, resultando em: $1,69 \mathrm{~kg} \mathrm{dm}^{-3}$;

nível 2: $1,78 \mathrm{~kg} \mathrm{dm}^{-3}$;

nível 3: $1,82 \mathrm{~kg} \mathrm{dm}^{-3}$.

A capacidade do vaso foi adotada como o conteúdo de água retirada do solo após sofrer saturação e consequente ação da gravidade, até o cessamento da drenagem, segundo Souza et al. (2000). A irrigação dos vasos foi controlada através do procedimento de pesagem diária, em balança de precisão entre 7 e $9 \mathrm{~h}$ da manhã para posterior reposição da água evapotranspirada no período, mantendo-se os vasos próximos à capacidade de campo, iniciada no $50^{\circ}$ dia após a estabilidade das mudas nos vasos.

A população de $M$. incognita foi obtida em plantas de canade-açúcar cultivadas em campo, mantida em casa de vegetação em tomateiro (Solanum lycopersicum L.) e posteriormente em pimentão (Capsicum annuum L.) em solo autoclavado. Antes do lançamento do experimento o inóculo foi multiplicado em plântulas de tomateiros com 20 dias de idade mantidas em vasos com mistura de solo, areia e húmus na proporção 3:1:1, respectivamente, devidamente esterilizado. Para o preparo do inóculo usou-se o método de Hussey \& Barker (1973) para extração de ovos a partir das raízes parasitadas do tomateiro e pimenteira. As inoculações foram efetuadas com pipetas de graduação automática sendo a suspensão vertida ao redor de cada planta. Cinco dias após a inoculação as plantas foram transferidas para vasos de $3 \mathrm{~L} \mathrm{e} 7 \mathrm{~cm}$ de diâmetro e mantidas em ambiente de casa de vegetação por mais 40 dias quando então foram extraídos os ovos para o experimento, conforme Hussey \& Barker (1973). As plantas de cana-de-açúcar foram inoculadas com suspensão de 6.000 ovos $\mathrm{mL}^{-1}$ planta $^{-1}$, após 50 dias de estabilidade das mudas micropropagadas. $\mathrm{O}$ inóculo foi vertido no solo com o auxílio de uma pipeta calibrada, em orifícios ao redor da planta. A colheita foi realizada aos 90 dias 
após a infestação do solo, o que corresponde a 120 dias após o transplante das plantas para os vasos.

Foram avaliados os seguintes parâmetros: altura da planta, diâmetro do colmo, número de folhas, matéria fresca das raízes, matéria fresca da parte aérea, matéria seca da parte aérea e número de ovos do nematoide por grama de raiz. Para a avaliação do crescimento morfológico as mensurações foram realizadas 120 dias após o transplante das plantas, com auxílio de trena e paquímetro. Para a avaliação da matéria seca as amostras coletadas foram armazenadas em sacos de papel previamente identificados e posteriormente pesadas e daí encaminhadas para secagem em estufa com circulação de ar forçada a $65^{\circ} \mathrm{C}$ por 72 $h$, e novamente pesadas.

Para a coleta dos ovos visando facilitar a remoção dos sistemas radiculares, os vasos foram colocados em um tanque de água, por alguns minutos e cuidadosamente o solo foi removido de cada planta de modo a minimizar as perdas de massa de ovos. Três lavagens de cada sistema radicular foram realizadas fazendo-as passar por baldes de água limpa tomando-se, em seguida, o peso da matéria fresca dos sistemas radiculares. Para extração dos ovos seguiu-se a técnica de Hussey \& Barker (1973). A estimativa populacional foi obtida através da contagem em caixas calibradas com auxílio de um microscópio óptico, em três repetições.

Os dados foram submetidos à análise de variância e, quando significativos, submetidos à análise de regressão utilizando-se os programas Excel e SAS.

\section{Resultados e Discussão}

Não foram significativos $(\mathrm{p}>0,05)$ os efeitos dos tratamentos no diâmetro do colmo, número de folhas, biomassa fresca das raízes e da parte aérea e a biomassa seca da parte aérea (Tabela 1), corroborando com Silva et al. (2006) que não obtiveram efeito significativo da densidade do solo $\left(1,0 ; 1,2 ; 1,4\right.$ e $\left.1,5 \mathrm{~kg} \mathrm{dm}^{-3}\right)$ sobre a produção de matéria seca da parte aérea das culturas de milho, soja, algodão e Brachiaria brizantha.

Resultados semelhantes também foram obtidos por Foloni et al. (2006), cujas densidades aplicadas $\left(1,1 ; 1,3\right.$ e 1,5 $\left.\mathrm{kg} \mathrm{dm}^{-3}\right)$ em solo de textura argilosa não interferiram no desenvolvimento nem na produção de biomassa seca dos adubos verde guandu, guandu anão e crotalaria, e da cultura da soja em casa de vegetação após 60 dias de cultivo. Segundo os autores, o aumento da densidade do solo em camadas subsuperficiais não é considerado fator limitante no crescimento aéreo das espécies estudadas confirmando, assim, os resultados da presente pesquisa uma vez que o crescimento das mudas de cana-de- açúcar, variedade RB 863129, não foi afetado pelos níveis de densidade do solo aplicados.

Efeitos significativos $(\mathrm{P} \leq 0,05)$ foram detectados para o comprimento do colmo e o número de ovos do nematoide. De acordo com Imhoff et al. (2000) a densidade máxima que a cultura suporta é de $1,70 \mathrm{~kg} \mathrm{dm}^{-3}$, o que corresponde a 1,70 $\mathrm{g} \mathrm{cm}^{-3}$ já que, para a cultura da cana-de-açúcar um aumento na densidade do solo, principalmente na camada superficial, prejudicaria o desenvolvimento do sistema radicular uma vez que a cultura tem o nível de exploração nutricional arranjado na camada arável do solo.

Densidades elevadas poderiam comprometer a respiração das raízes e exercer resistência ao seu desenvolvimento; entretanto, a densidade de $1,82 \mathrm{~kg} \mathrm{dm}^{-3}$ não afetou, neste estudo, a quase totalidade das variáveis de desenvolvimento da planta avaliadas corroborando com Freddi et al. (2009) que observaram, trabalhando com a cultura do milho em solo compactado, que as características agronômicas da cultura não foram afetadas apresentando desenvolvimento homogêneo.

No presente estudo, Figura 1, a altura do colmo obteve maior média $(74,50 \mathrm{~cm})$ quando submetida ao maior nível de densidade do solo (64\%), representado por regressão linear $\left(\mathrm{R}^{2}=72,9 \%\right)$, corroborando com Bordin et al. (2005) que constataram aumento na altura de mudas de acerola propagadas por estaquia (de 10,9 para $50,3 \mathrm{~cm}$ ) à medida que foi aumentado o nível de compactação no solo (de 1,0 para $1,6 \mathrm{~kg} \mathrm{dm}^{-3}$, respectivamente), concluindo que o aumento da densidade do solo não impediu o desenvolvimento da parte aérea nem a penetração das raízes das mudas da aceroleira. Resultados contrários foram observados por Cordeiro Neto (2012) que, estudando o comportamento de duas variedades

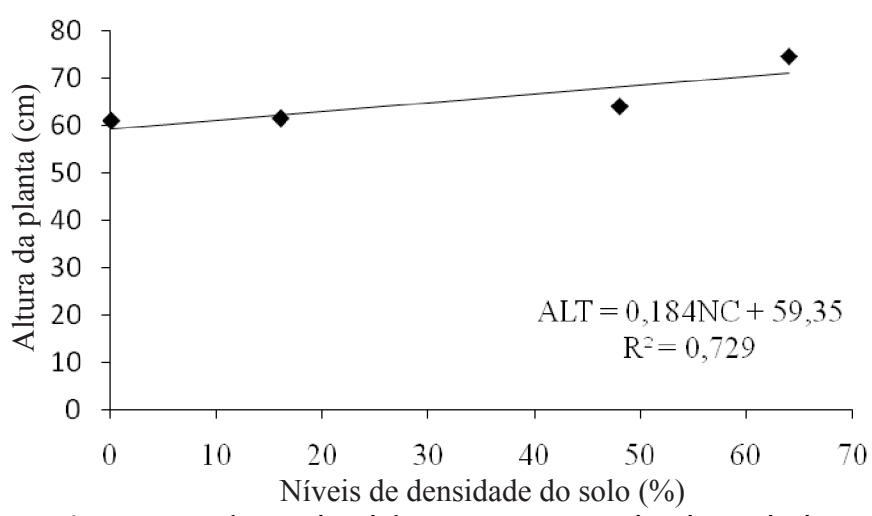

Figura 1. Efeito de diferentes níveis de densidade do solo (NC) na altura do colmo (ALT), em casa de vegetação, 120 dias após o transplante das mudas

Tabela 1. Quadrado médio das variáveis biométricas da cana-de-açúcar (diâmetro do colmo (DC), altura do colmo (AC), número de folhas (NF), matéria fresca da parte aérea (MFPA), matéria fresca da raiz (MFR), matéria seca da parte aérea (MSPA)) e do número de ovos de Meloidogyne incognita por planta (OP), 120 dias após o transplante das mudas

\begin{tabular}{lcccccccc}
\hline Fonte de & \multirow{2}{*}{ GL } & \multicolumn{9}{c}{ Quadrado médio } \\
\cline { 3 - 9 } variação & & DC & AC & NF & MFPA & MFR & MSPA & OP \\
Tratamento & 4 & $0,011^{\text {ns }}$ & $430,03^{*}$ & $0,069^{\text {ns }}$ & $3,87^{\text {ns }}$ & $0,87^{\text {ns }}$ & $0,69^{\text {ns }}$ & $16,06^{*}$ \\
Resíduo & 19 & 0,009 & 94,19 & 0,113 & 3,69 & 0,84 & 0,75 & 0,017 \\
CV (\%) & - & 8,120 & 15,51 & 12,490 & 18,96 & 17,95 & 19,41 & 4,140 \\
\hline
\end{tabular}

* significativo a nível de 0,05 de probabilidade; ns - não significativo pelo teste $\mathrm{F}$ 
de cana-de-açúcar (RB962962 e RB863129) em três diferentes níveis de compactação $\left(1,5 ; 1,6\right.$ e $\left.1,7 \mathrm{~kg} \mathrm{dm}^{-3}\right)$, não encontrou diferença significativa na altura das plantas; mesmo assim, Silva et al. (2006) verificaram influência da compactação do solo na altura de plantas de soja, que foi maior $(94,81 \%)$ na maior densidade aplicada $\left(1,5 \mathrm{~kg} \mathrm{dm}^{-3}\right)$.

Contrários aos resultados obtidos neste estudo, Freddi et al. (2009) verificaram, estudando cultivares de milho sob solo compactado, que as características agronômicas foram afetadas porém a compactação superficial não afetou a produção total de raízes embora o impedimento físico tenha alterado a distribuição do sistema radicular das plantas. De acordo com Silva et al. (2006) a resistência do solo à penetração é uma das propriedades físicas diretamente relacionadas com o crescimento das plantas e modificadas pelos sistemas de preparo do solo. Valores excessivos de resistência do solo podem influenciar o crescimento das raízes, em comprimento e diâmetro.

A compactação do solo afetou a reprodução do nematoide que diminuiu à medida que houve aumento da compactação (Figura 2), resultados contrários aos de Cordeiro Neto (2012) que não observou influência das variedades de cana-de-açúcar (RB962962 e RB863129) nem níveis de compactação do solo (1,5; 1,6 e 1,7 kg dm$\left.{ }^{-3}\right)$ na reprodução de $M$. incognita. Segundo Cardoso et al. (2012) o gênero Meloidogyne sp. se relaciona positivamente com o espaço poroso do solo de vez que é uma espécie que necessita essencialmente de trocas gasosas através de sua cutícula sendo assim predominante em solos arenosos.

De acordo com Ribeiro et al. (2009) e Vicente (2011) a maior densidade populacional de nematoides se concentra na camada superficial do solo na qual se encontram as primeiras camadas do sistema radicular, o que também pode estar associado à compactação das camadas mais profundas haja vista que modificam os espaços porosos, diminuem a oxigenação do solo e a quantidade de nutrientes.

Resultados semelhantes foram encontrados por Lanza et al. (2004) trabalhando com sobrevivência da população do fungo Metarhizium anisopliae em três tipos de solo (textura argilosa, média e arenosa média) com diferentes níveis de compactação, segundo os quais o impacto da compactação foi mais acentuado no solo de textura média com ocorrência de queda mais

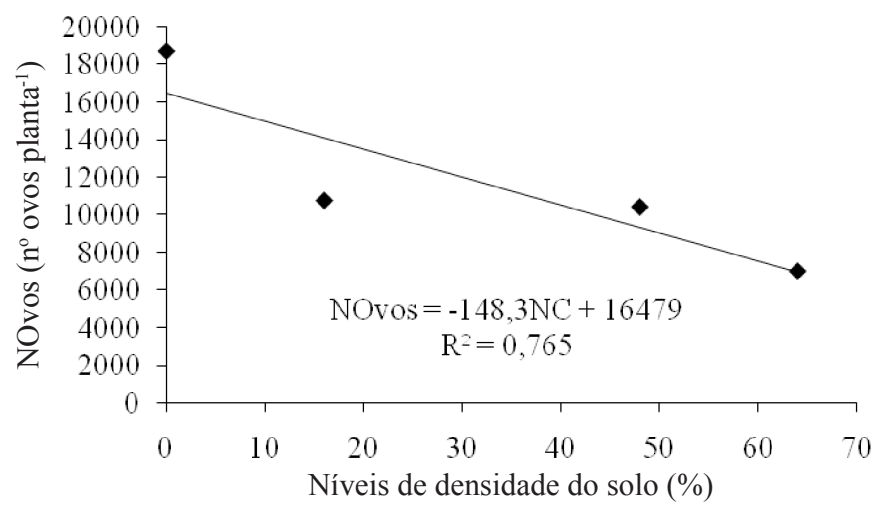

Figura 2. Efeito de diferentes níveis de densidade do solo na reprodução de Meloidogyne incognita em cana-de-açúcar, 90 dias após a infestação do solo acentuada na sobrevivência do fungo e em todos os níveis de compactação aplicados $\left(1,22 ; 1,44\right.$ e 1,65 $\left.\mathrm{g} \mathrm{cm}^{-3}\right)$.

De acordo com Miranda et al. (2012) a densidade do solo é crítica para a mobilidade e atividade dos nematoides mas, quando muito alta, afeta a sobrevivência devido às limitações de oxigênio. Vicente (2011) relata que a densidade populacional de nematoides está diretamente relacionada com a quantidade de matéria orgânica no solo de vez que esta fornece maior mobilidade e oxigenação para esses organismos, corroborando com Olabiyi et al. (2009) que afirmam que os parasitos de plantas são favorecidos em solos com textura arenosa.

Ademais, embora Li et al. (2007) afirmem que a distribuição de nematoides parasitas de plantas no perfil do solo seja reflexo da distribuição das raízes, os resultados obtidos no presente estudo não evidenciaram alterações na matéria fresca nem seca das raízes, nos diferentes níveis de compactação estudados ou entre os tratamentos com nematoide e a testemunha absoluta (sem nematoide) (Tabela 1). É provável que a rusticidade da cana-de-açúcar tenha favorecido a tolerância da planta aos estresses aplicados (compactação do solo e nematoide) que se apresenta em menor severidade em condições controladas de casa de vegetação do que em condições de campo às quais vários fatores de estresse geralmente estão envolvidos (Li et al., 2007; Kimenju et al., 2009; Mondino et al., 2009; Cardoso et al., 2012).

Por outro lado e como parasitas obrigados, os nematoides do gênero Meloidogyne apresentam relação balanceada e complexa com a planta hospedeira. Por exemplo, a duração do ciclo, que pode variar de três semanas a vários meses, é função de uma série de fatores destacando-se condições do solo e da planta (David \& Triantaphyllou, 1967; Pedrosa et al., 1996). O raio sexual também é influenciado pelas condições ambientais; os machos são mais numerosos em altas infestações quando há limitação de espaço e competição por alimento dentro da raiz, em más hospedeiras e em plantas debilitadas ou velhas (David \& Triantaphyllou, 1967; Pedrosa et al., 1996). É possível, então, especular que a diminuição da densidade populacional de M. incognita com o aumento da compactação do solo esteja associada à limitação de espaço, redução da oxigenação do solo e ao aumento do número de machos e do comprimento do ciclo de vida do nematoide, diminuindo a taxa reprodutiva.

\section{Conclusão}

O aumento dos níveis de densidade do solo de 1,65 para 1,82 $\mathrm{kg} \mathrm{dm}^{-3}$ afetou o comprimento do colmo, da cana-de-açúcar variedade RB863129; contudo, reduziu a multiplicação de $M$. incognita.

\section{Agradecimentos}

À FACEPE, pelo financiamento da pesquisa.

\section{Literatura Citada}

ABNT - Associação Brasileira de Normas Técnicas. NBR 7182: Solo. Ensaio de compactação - ABNT. 1986. NBR 3. Rio de Janeiro: ABNT, 1986. 11p. 
Bordin, I.; Neves, C. S. V. J.; Azevedo, M. C. B.; Vidal, L. H. I. Desenvolvimento de mudas de aceroleira propagadas por estacas e sementes em solo compactado. Ciência Rural, v.35, p.530-536, 2005.

Campos, A. P.; Vale, D. W.; Araújo, E. S.; Corradi, M. M.; Yamauti, M. S.; Fernandes, O. A.; Freitas. S. Manejo integrado de pragas. Jaboticabal: FUNEP, 2006. p.59-80.

Cardoso, M. O; Pedrosa, E. M. R.; Rolim, M. M.; Silva, E. F. F.; Barros, P. A. Effects of soil mechanical resistance on nematode community structure under conventional sugarcane and remaining of Atlantic Forest. Environmental Monitoring and Assessment, v.184, p.3529-3544, 2012.

Cordeiro Neto, A. T. Efeito da compactação do solo e da vinhaça no desenvolvimento inicial e nutrição da cana-de-açúcar parasitada por nematóides. Recife: UFRPE, 2012. 67p. Dissertação Mestrado

David, R. G.; Triantaphyllou, A. C. Influence of the environment and sex differentiation of root-knot nematodes. I. Effect of infection density, age of the host plant and soil temperature. Nematologica, v.13, p.102-110,1967.

EMBRAPA - Empresa Brasileira de Pesquisa Agropecuária. Centro Nacional de Pesquisa de Solos. Sistema brasileiro de classificação de solos. Rio de Janeiro: EMBRAPA, 1999. 412p.

Freddi, O. S.; Centurion, J. F.; Duarte, A. P.; Leonel, C. L. Compactação do solo e produção de cultivares de milho em Latossolo vermelho. I - características de planta, solo e índice S. Revista Brasileira de Ciência do Solo, v.33, p.793-803, 2009.

Foloni, J. S. S.; Lima, S. L.; Bull, L. T. Crescimento aéreo e radicular da soja e de plantas de cobertura em camadas compactadas de solo. Revista Brasileira de Ciência do Solo, v.30, p.49-56, 2006

Hussey, R. S.; Barker, K. R. A comparison of methods of collecting inocula for Meloidogyne spp., including a new technique. Plant Disease Reporter, v.57, p.1025-1028, 1973.

Iaia, A. M.; Maia, J. C. S.; Kim, M. E. Uso do penetrômetro eletrônico na avaliação da resistência do solo cultivado com cana-de-açúcar. Revista Brasileira de Engenharia Agrícola e Ambiental, v.10, p.523-530, 2006.

Imhoff, S.; Silva, A. P.; Tormena, C. A. Aplicações da curva de resistência no controle da qualidade física de um solo sob pastagem. Pesquisa Agropecuária Brasileira, v.35, p.14931500, 2000.

Kimenju, J. W.; Karanja, N. K.; Mutua, G. K.; Rimberia, B. M.; Wachira, P. M. Nematode community structure as influenced by land use and intensity of cultivation. Tropical and Subtropical Agroecossystems, v.11, p.353-360, 2009.

Lanza, L. M.; Monteiro, A. C.; Malheiros, E. B. População de Metarhizium anisopliae em diferentes tipos e graus de compactação de solo. Ciência Rural, v.34, p.1757-1762, 2004.

Li, Q.; Liang, W.; Ou, W. Responses of nematode communities to different land uses in an aquatic brown soil. Biodiversity Science, v.15, p.172-179, 2007.

Matos, D. S. S.; Pedrosa, E. M. R.; Guimarães, L. M. P., Silva, C. V. M. A.; Barbosa, N. M. R. Relações entre a nematofauna e atributos químicos de solo com vinhaça. Nematropica, v.41, p.1-9, 2011.
Medeiros, R. D.; Soares, A. A.; Guimarães, R. M. Compactação do solo e manejo da água. I: Efeitos sobre a absorção de N, $\mathrm{P}, \mathrm{K}$, massa seca de raízes e parte aérea das plantas de arroz. Ciência e Agrotecnologia, v.29, p.940-947, 2005.

Miranda, L. L. D.; Gil, M. A.; MenegattI, C. C. Danos causados por nematóides a variedades de cana-de-açúcar em cana planta. Nematologia Brasileira, v.27, p.69-73, 2003.

Miranda, T. L; Pedrosa, E. M. R., Silva, E. F. F., Rolim, M. M. Alterações físicas e biológicas em solo cultivado com canade-açúcar após colheita e aplicação de vinhaça. Agrária, v.7, p.150-158, 2012.

Mondino, E. A.; Tavares, O. C. H.; Ebeling, A. G.; Figueira, A. F; Quintero, E. I.; Berbara, R. L. L. Avaliação das comunidades de nematóides do solo em agroecossistemas orgânicos. ActaScientiarum Agronomy, v.31, p.509-515, 2009.

Olabiyi, T. I.; Olayiwola, A. O.; Oyediran, G. O. Influence of soil textures on distribution of phytonematodes in the South Western.World Journal of Agricultural Sciences, v.5, p.557-560, 2009.

Pattison, A. B.; Moody, P. W.; Badcock, K. A.; Smith, L. J.; Armour, J. A.; Rasiah, V.; Cobon, J. A.; Gulino, L. M.; Mayer, R. Development of key soil health indicators for the Australian banana industry. Applied Soil Ecology, v.40, p.155-164, 2008.

Pedrosa, E. M. R.; Hussey, R. S.; Boerma, H. R. Penetration and post-infectional development and reproduction of Meloidogyne arenaria races 1 and 2 on susceptible and resistant soybean genotypes. Journal of Nematology, v.28, p.343-351, 1996.

Pedrosa, E. M. R.; Rolim, M. M.; Albuquerque, P. H. S.; Cunha, A. C. Supressividade de nematóides em cana-de-açúcar por adição de vinhaça ao solo. Revista Brasileira de Engenharia Agrícola e Ambiental, v.9, p.197-201, 2005.

Ribeiro, C. F. R.; Xavier, F. R. P.; Xavier, A. A.; Almeida, V. F; Mizobutsi, E. H.; Campos, V. P.; Ferraz, S.; Dias-Arieira, C. R. Flutuação populacional e efeito da distância e profundidade sobre nematóides em bananeira no norte de Minas Gerais. Revista Brasileira de Fruticultura, v.31, p.103-111, 2009.

Silva, G. J.; Maia, J. C. S.; Bianchini, A. Crescimento da parte aérea de plantas cultivadas em vaso, submetidas a irrigação subsuperficial e a diferentes graus de compactação de um Latossolo vermelho-escuro distrófico. Revista Brasileira de Ciências do Solo, v.30, p.31-40, 2006.

Souza, C. C.; Oliveira, F. A.; Silva, I. F.; Amorim Neto, M. S. Avaliação de métodos de determinação de água disponível e manejo da irrigação em terra roxa sob cultivo de algodoeiro herbáceo. Revista Brasileira de Engenharia Agrícola e Ambiental, v.4, p.338-342, 2000.

UFC - Universidade Federal do Ceará. Recomendações de adubação e calagem para o Estado do Ceará. In: Ceará. Fortaleza: UFC/CCA, 1993. 248p.

Vicente, T. F. S. Estabilidade de agregados em relações de atributos do solo com a nematofauna em áreas de cultivo de cana-de-açúcar. Recife: UFRPE, 2011. 84p. Dissertação Mestrado 